Measuring the effects of caffeine and L-theanine on cognitive performance: a protocol for self-directed, mobile n-of-1 studies

Eddye Golden, MPH1*, Matthew Johnson, MSc1, Michael Jones, MSc1, Ryan Viglizzo, MPS1, Jason Bobe, MSc1*, Noah Zimmerman, PhD1 IInstitute for Next Generation Healthcare, Department of Genetics and Genomic Sciences, Icahn

School of Medicine at Mount Sinai, New York, NY, USA

Correspondence:

Jason Bobe

Keywords: n-of-1 trials1, cognition2, digital health3, caffeine4, nootropics5, cognitive6, mobile app 7 , mhealth8

Word Count: 4982 including abstract and contribution statements (excluding appendices)

\title{
Abstract
}

Background:

The growing consumer digital tools market has made using individual health data to inform lifestyle changes more accessible than ever. The n-of-1 trial -- a single participant, multiple crossover, comparative effectiveness trial -- offers methodological tools that link interventions directly with personalized outcomes to determine the best treatment for an individual. We have developed a complete digital platform to support self-directed n-of-1 trials, comprised of virtual study on-boarding, visual informed consent, device integrations, in-app assessments, and automated data analysis.

Objective:

To evaluate the n-of-1 platform, a pilot study was launched to investigate the effects of commonly consumed substances on cognition. The purpose of the study is to allow an individual to measure the effect of 2 treatments (caffeine alone vs. caffeine + L-theanine) on 3 measures of cognitive performance: creative thinking, processing speed, and visual attention. Upon completion of the study, individuals receive personalized results that compare the impact of the two treatments on each of the cognitive performance measures.

Methods: After the onboarding process, participants are randomized to a study length $(5,15$, or 27 days), starting treatment (caffeine or caffeine + L-theanine), and app notification frequency (light, 


\section{Measuring the effects of caffeine and L-theanine on cognitive performance: a protocol for}

self-directed, mobile n-of-1 studies

40 moderate). Each trial begins with a baseline period, during which participants abstain from either

41 treatment, followed by 2 randomized counterbalanced treatment sequences (either ABBA or

42 BAAB). Throughout the trial, daily tests assess participant cognitive performance. These tests

43 are digital versions of the Remote Associates Test, Stroop Test, and Trail Making Test, and are

44 implemented directly in the n-of-1 mobile application ("N1"). Assessments are completed at a

45 fixed time, defined by the individual during study setup. Treatments are taken daily within a

46 fixed time window prior to the user-defined assessment time.

47

48 Results:

49 Cognitive assessment results are analyzed using a linear model with factors for treatment and

50 block, and each treatment is compared to baseline. We launched our N1 app on the Apple App

51 Store in October 2019 and have recruited over 40 participants to date.

52

53 Conclusion:

54 This platform provides individuals the opportunity to investigate their response to treatments

55 through n-of-1 methods, empowering them to make data-driven, personalized lifestyle choices. 


\section{Measuring the effects of caffeine and L-theanine on cognitive performance: a protocol for}

self-directed, mobile n-of-1 studies

\section{$1 \quad$ Introduction}

57 Technological and medical innovation have brought about a rapid digitalization of research.

58 Digital research has conceptually grown to incorporate a broad set of medical and scientific

59 themes -- genomics, AI, wearable technology, mobile apps, longitudinal data capture, among

60 others. With this digitalization of medical research, we see increased interest in digital

61 biomarkers, as well as the growing practice and value of longitudinal data capture, electronic

62 patient reported outcomes, and effective validation strategies for digital health tools (1)(2).

The growing availability of digital research tools creates a unique opportunity to further develop health research. Individuals are now presented with increased opportunities to participate remotely in research, including going through informed consent processes and interacting with study teams via digital platforms. Significant components of research, such as informed consent and study team communications, are being digitized to enhance user experience, comprehension, and accessibility (3)(4). Using these methods, some digital studies are able to enroll large numbers of participants in a short time. We see this at play in the app-based mPower study, which enrolled more than 10,000 participants in the first year (5), and the Apple Heart Study, in which Stanford researchers enrolled over 400,000 individuals in one year for a remote, singlearm study using the Apple Watch to identify atrial flutter (6). Apple also recently launched Apple Research, an app designed to better streamline the enrollment and management of mobile health studies (7). These trends open up the digital research space to the concept of the n-of-1 trial.

An n-of-1 trial monitors the effects of different treatments or interventions on a single participant, where $n=1$. It is typically structured as a single-patient, multiple-crossover comparative effectiveness trial. Each participant tests 1 or more interventions multiple times over the course of the trial, and subsequently compares the outcomes of those interventions (8). N-of1 trials have been used in healthcare when a clinician wants to test different medications, dosages, or treatments on a patient to determine individual response, and thus craft a personalized and effective route to health. The n-of- 1 trial is particularly useful where limited evidence exists for a particular treatment or outcome, or where there is variability across individuals in treatment response (8). The success of this clinical implementation is dependent on the study design (what is being compared), and the willingness and collaboration of the patient, and the capacity of the clinician to design and implement an n-of-1 trial. With more than 2000 of these trials published to date, examples of previous implementations include a post-meal activity assessment for lowering postprandial glycaemia among people with type 2 diabetes (9), as well as stimulant effectiveness among people with attention deficit/hyperactivity disorder (10). design, conduct, and analyze methodologically sound, statistically robust n-of-1 trials. We are 


\section{Measuring the effects of caffeine and L-theanine on cognitive performance: a protocol for}

self-directed, mobile n-of-1 studies

performance) and to interventions (caffeine versus caffeine + L-theanine) that are fast-acting, controllable, and easily measurable. Each individual will participate in his/her own study, with treatments applied in sequence to assess whether L-theanine has a cognitive effect beyond that of caffeine alone for that person. This design choice allows us to use adapted versions of validated cognitive instruments readily available in the NIH Toolbox and Apple ResearchKit, and individuals may engage in interventions that are already part of their daily lives (e.g. drinking coffee or tea) (11)(12). These novel methods and tools are designed to empower individuals to make more rational, data-driven choices about their own health and wellness. This implementation will also allow us to assess the effectiveness of the n-of- 1 trial within the current digital research landscape.

\section{Methods}

\subsection{Study design}

We designed and developed a smartphone app and software platform that provides individuals the opportunity to remotely engage in personalized n-of- 1 investigations. The platform facilitates enrollment, longitudinal data capture, digital biomarker measurement, administration of validated instruments, study task notifications and reminders, statistical analysis, and access to data and results. To evaluate the n-of-1 platform, we aimed to design a study that is relevant to broad audiences, incorporates ubiquitous and safe treatments, and utilizes validated assessment instruments in a digital format. A cognition study that evaluates the effects of two commonly consumed substances, caffeine and L-theanine, meet these criteria and serves as our first study deployed on the platform.

\subsubsection{Treatments}

We will measure the effects of two different treatments on daily cognitive function:

- Treatment A: caffeine (50-400 mg, based on choice of beverage/supplement)

- Treatment B: caffeine (50-400 mg, based on choice of beverage/supplement) + Ltheanine (approximately $250 \mathrm{mg}$, based on choice of beverage/supplement)

These treatments were chosen due to their ubiquity, common daily use, efficacy, and safety. Participants may choose two beverages (e.g. coffee and tea) or they may also use an over-the-counter supplement (e.g. caffeine pills or L-theanine pills). Caffeine is one of the world's most commonly consumed drugs, and is often used to improve alertness and response time (13). L-theanine is an amino acid derived from tea leaves that is believed to have calming physical effects when consumed (14). It is found most commonly in green tea and other teas, or in supplement form. The FDA has given L-theanine Generally Recognized as Safe ("GRAS") status (15). Studies suggest that L-theanine may improve cognitive performance, and it is claimed that the combination of L-theanine with caffeine allows the consumer to feel the positive cognitive effects of caffeine while counteracting 
Measuring the effects of caffeine and L-theanine on cognitive performance: a protocol for self-directed, mobile n-of-1 studies

the "jitters," and reducing "mind wandering" (16). The dosages vary between beverage and supplement choices, and so we provide a reference range to participants based on commonly consumed caffeinated drinks, available supplement dosages, and FDA compound review recommendations (15)(17).

\subsubsection{Treatment Blocks}

We employ a randomized counterbalanced treatment design for each study length (ABBA or BAAB) (8). Participants are randomized into 1 of 3 study lengths -5 days, 15 days, and 27 days. Three different study lengths (short, medium, and long) are used in order to assess the effect of trial length on adherence and attrition. The treatment periods are either 1 day, 3 days, or 5 days, depending on study length.

Our 5-day study includes 1 day of baseline, 2 days of treatment A (caffeine) and 2 days of treatment B (caffeine + L-theanine), with treatment periods lasting 1 day. With "N" as baseline, a participant may be randomized into a 5-day study of either of the following patterns:

$\mathrm{N}+(\mathrm{A})+(\mathrm{B})+(\mathrm{B})+(\mathrm{A})$

$\mathrm{N}+(\mathrm{B})+(\mathrm{A})+(\mathrm{A})+(\mathrm{B})$

Our 15-day study includes 3 days of baseline, 6 days of treatment A, and 6 days of treatment $\mathrm{B}$, with treatment periods lasting 3 days. As such, a participant may be randomized into a 15-day study of either of the following patterns:

$\mathrm{NNN}+(\mathrm{AAA})+(\mathrm{BBB})+(\mathrm{BBB})+(\mathrm{AAA})$

$\mathrm{NNN}+(\mathrm{BBB})+(\mathrm{AAA})+(\mathrm{AAA})+(\mathrm{BBB})$

Our 27-day study includes 7 days of baseline, 10 days of treatment A, and 10 days of treatment $\mathrm{B}$, with treatment periods lasting 5 days. Participants may be randomized into one of the following 27-day studies:

NNNNNNN + (AAAAA) $+($ BBBBB $)+($ BBBBB $)+($ AAAAA $)$

NNNNNNN + (BBBBB $)+($ AAAAA $)+($ AAAAA $)+($ BBBBB $)$

Participants must complete $1 / 3$ of treatments and assessments during each treatment period to avoid study failure. The 5-day study is too short for a participant to miss any days of treatment/assessment and still have sufficient data to calculate a result. Individuals in the 15- and 27-day studies can miss up to 2 days per treatment period before study failure.

\subsubsection{Primary Outcome Measures}

To assess cognitive function, we use three validated instruments adapted from the NIH Toolbox and implemented with Apple's ResearchKit: 
2.1.3.1 Remote Associates Test (RAT), a measure of creative thinking (Fig. 1). The RAT measures an individual's "creative" cognition by presenting them with a word problem consisting of 3 stimulus words and asking them to propose a fourth solution word that ties them together. For example, an individual may be prompted with the following: "sleeping, bean, trash." They would then try to come up with a linking fourth term, which in this case is "bag." It has been shown that problem solvers' success on items from the original RAT reliably correlates with their success on classic insight problems (18)(19)(20)(21). This test was implemented as a variation of the original RAT, developed as a custom digital ActiveTask within Apple's ResearchKit framework.

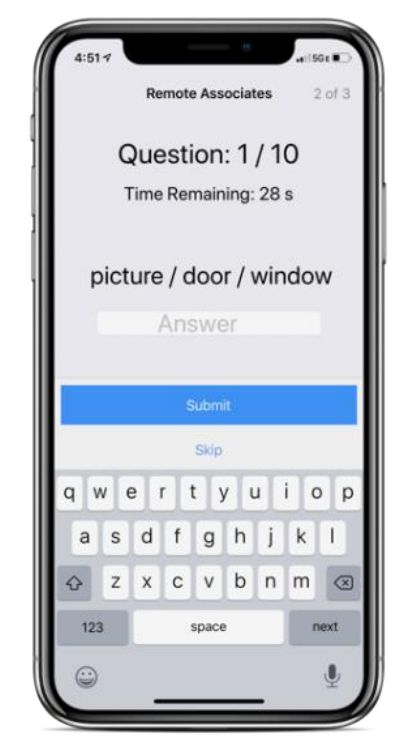

Figure 1 | Sample of Remote Associates Test

Six metrics are collected in our implementation of the RAT: words presented, word difficulty level, participant response/answer, average response time (in seconds), and score percentage (correct answers out of a possible 10) (22). While multiple metrics are collected in our implementation of the RAT, only score percentage is analyzed and relayed back as an end result to the participant. Further details regarding our custom implementation of the RAT can be found in Appendix A.

2.1.3.2 Stroop Test, a measure of selective attention and processing speed. (Fig. 2)

The Stroop Test is a measurement of executive function/reaction time. It assesses the ability of the user to distinguish between a printed word that names a color 
and the color of the actual text (23). We use an abridged version of the test available from Apple ResearchKit as a predefined ActiveTask. In our abridged mobile version, a single task is presented and we record a metric that captures both accuracy and reaction speed, known as the rate corrected score (24)(25). Additional details about the original and Apple-implemented versions of the
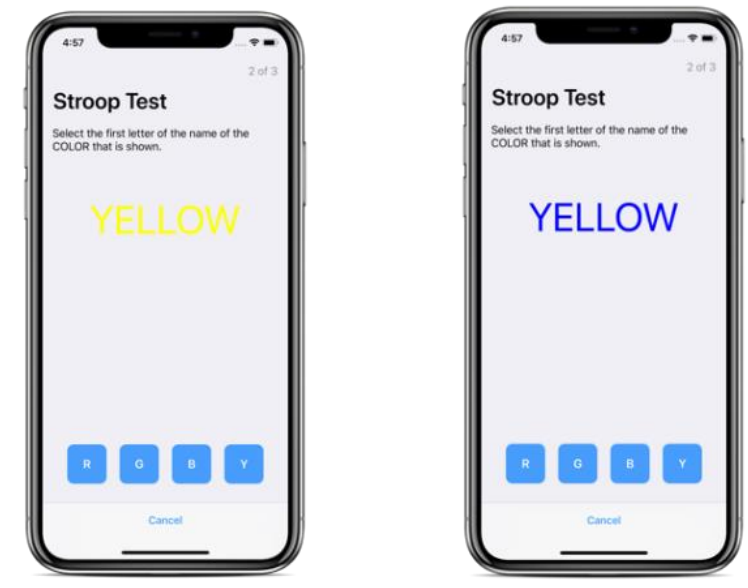

Figure 2 | Samples of Stroop Test

2.1.3.3 Trail Making Test (TMT), a measure of visual attention and taskswitching. (Fig. 3)

The TMT is a standard component of many neuropsychological batteries and is one of the most commonly used tests because of its high sensitivity to the presence of cognitive impairment (26)(27)(28)(29).

This test is implemented as a predefined ActiveTask through Apple's ResearchKit. As part of this predefined task, 13 dots are presented by default rather than 25 as in the original test. In this implementation, a line is drawn automatically as participants tap the next labeled dot in ascending order (i.e. when a participant taps " 1 " and subsequently taps " $\mathrm{A}$ ", a line is drawn between the two dots.) For this implementation, only Part B of the test is presented to reduce the time commitment required for the participant. Part B is the more difficult of the two parts of the original test and there is evidence that Part B performance is indicative of executive function, where the difficulty of the task may reflect the cognitive flexibility of shifting the course of an ongoing activity (30)(29)(31). 


\subsection{Inclusion \& Exclusion Criteria}

Eligibility of potential participants is determined through a digital eligibility screener. United States. contraindication to caffeine. These exclusion criteria are based on the potential negative health effects of caffeine (32). Before joining the study, we advise individuals to consult a medical professional if they are unsure of how caffeine may affect them. Due to the ongoing and individual nature of the study, users may enroll at a later date if they are currently ineligible (e.g. due to pregnancy status or age).

We developed our inclusion and exclusion criteria in an attempt to exclude as few potential participants as possible, with primary consideration for health and safety. While certain assessments may pose challenges to certain populations (e.g. RAT to non-native English speakers, and Stroop Test to colorblind individuals), we have decided not to exclude these populations because they may still receive study results based on significant outcomes from one of the cognitive tests. Each test outcome is analyzed separately, so a treatment outcome is possible where focus is given to only one test in the cognitive assessment. Additionally, since a person serves as their own control in an n-of-1 trial, other possibly disadvantageous factors, such as mild cognitive impairment, should not matter in achieving a significant result. 
Measuring the effects of caffeine and L-theanine on cognitive performance: a protocol for self-directed, mobile n-of-1 studies

\subsection{Informed Consent}

After a user downloads the app, they register for an account and go through a study onboarding process. The onboarding includes a study-specific eligibility screener, a brief introduction to nof- 1 studies, and a digital informed consent process.

Our informed consent process is modeled on Sage Bionetworks' multimedia eConsent framework (33). It includes a short, self-guided digital consent module that clearly presents screens outlining the following parts of the consent form: study procedures, data privacy and security, data sharing, benefits, risks, withdrawal process, and consent review. (Fig. 4)
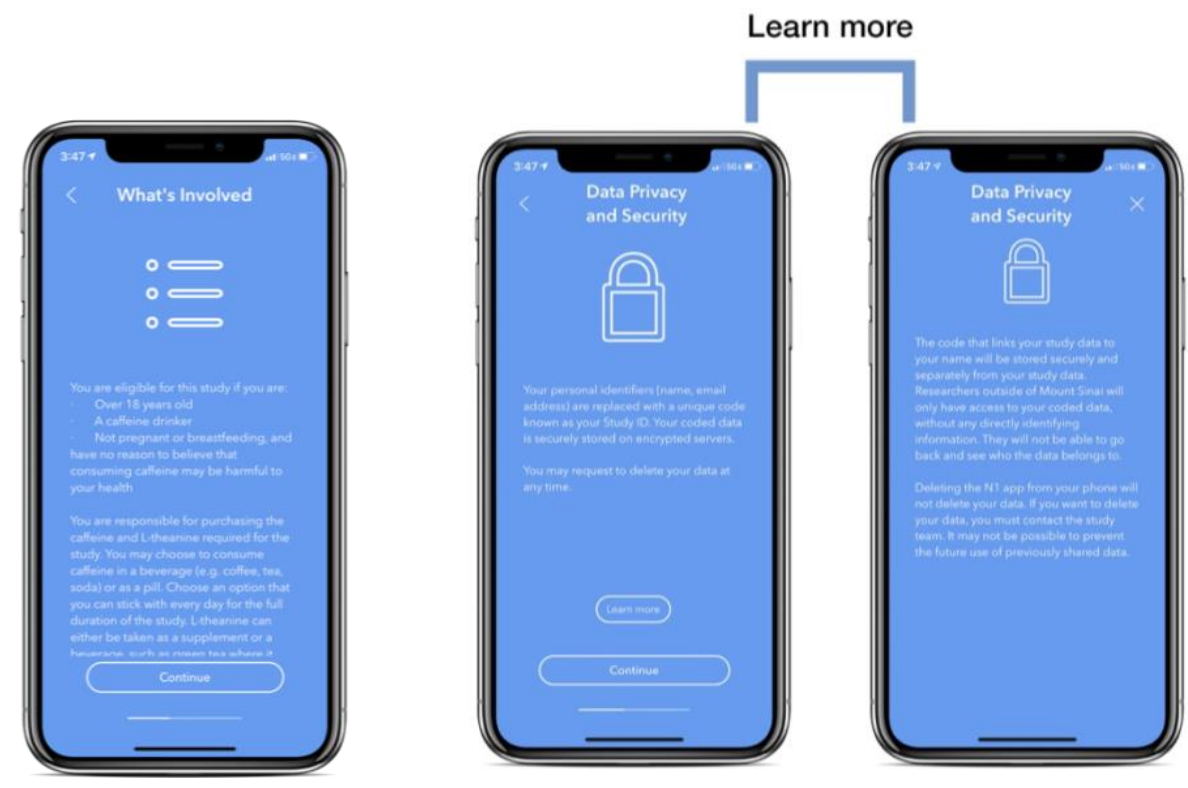

Figure 4 | Sample screens from the digital consent module

\subsection{Procedures}

After the consent module, the participant is presented with a PDF version of the full consent document. Participants type their name and provide an electronic signature after reviewing the full consent form. Their signature and timestamp are digitally placed on the consent and the signed version is subsequently available to the user for viewing within the study app at any time. Currently, informed consent is available in English only. The participant is provided the option of contacting the research staff during regular business hours if they have questions about the consent form or the study. the study app, participants engage in a study that is randomized by study length $(5,15$, or 27 days), treatment sequence (starting with treatment A or B), and app notification frequency 


\section{Measuring the effects of caffeine and L-theanine on cognitive performance: a protocol for}

self-directed, mobile n-of-1 studies

(moderate or light). Participants log their choice of beverages or supplements for the study and choose a daily fixed time at which to measure their cognitive performance via the app. They are instructed to consume their treatment 1 hour prior to the cognitive assessment and are given a reminder 30-minutes prior to the start of the assessment window. Participants are given a 2-hour window in which to complete the cognitive assessment (Fig 5). Participants receive daily reminders via notifications in the app to take their treatments and complete the cognitive assessment. The daily cognitive assessment takes approximately 5 minutes total to complete.

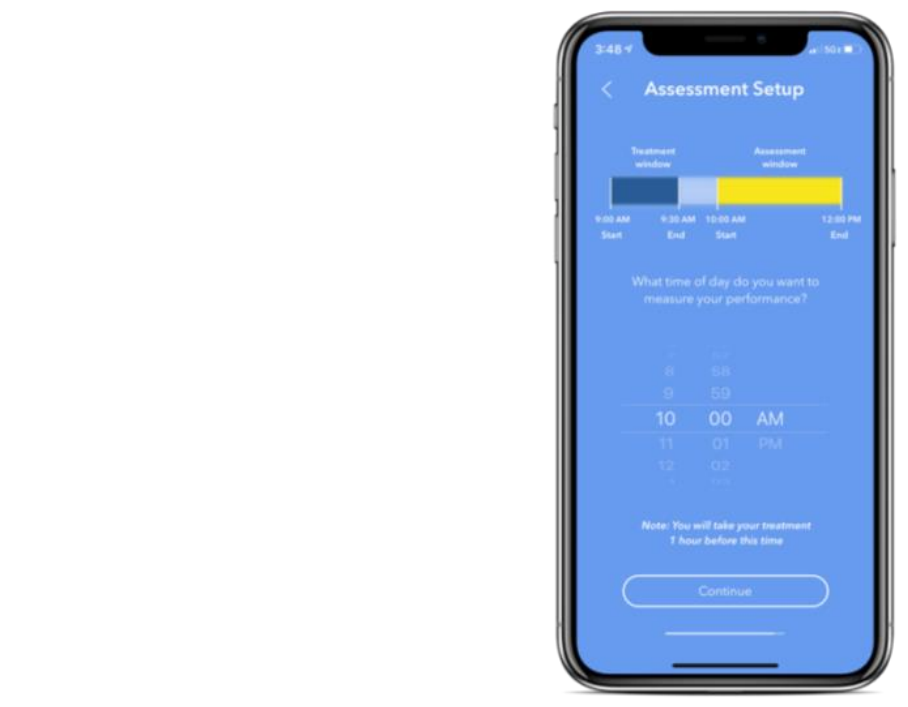

Figure 5 | Screenshot of assessment setup and treatment/assessment windows

The trial begins with a baseline period, where participants take the daily cognitive assessment but do not take either treatment. Participants then alternate between treatment A and treatment B for the duration of the study, according to the instructions in the app. We employ a randomized counterbalanced design for each participant's study (either ABBA or BAAB) (8). Participants may $\log$ any daily occurrences related to the study, such as missed treatments, changed beverages, or interruptions that may have impacted their performance on the cognitive assessments. Numerous additional data categories and variables are collected during the study (See Appendix B). To mitigate the risk of influencing the study outcomes, the scores of daily cognitive assessments are not returned at the time of completion. Instead, individual study results are processed and provided to the participant at the conclusion of the study.

To improve the likelihood that individuals are able to obtain "actionable results", we also invite participants to enroll in a longer study after completing their initial study. While this feature is aimed at users initially enrolled in the 5-day study because they are the most likely group to obtain a statistically inconclusive result, it will be made available to all users who have completed a study. If the participant wants to continue, they will be able to choose their subsequent study length from the 3 available study lengths (5, 15, and 27 days). Currently, data 


\section{Measuring the effects of caffeine and L-theanine on cognitive performance: a protocol for}

self-directed, mobile n-of-1 studies

from multiple studies will not be grouped and will be analyzed as independent studies. We anticipate adding a "grouped analysis" feature in the future.

\subsection{Recruitment}

Our N1 app is available for download in the Apple App Store. To ensure a diverse sample, the app and cognition study will be promoted using Mount Sinai Health System recruitment channels, related conferences (e.g. Quantified Self conference), and through promotional messaging on online message boards and social media (e.g. Reddit, Twitter, Facebook).

Recruitment is largely targeted to individuals who have shown interest in and/or previous experience using L-theanine and similar compounds through posting on specific L-theaninerelated web pages and social media groups. However, people may join our study without prior experience or knowledge of L-theanine, as recruitment will also include more general websites. Our recruitment strategy is broad, with no population-based restrictions.

\subsubsection{Safety Monitoring}

Considering this is an online, remote, and individualized study, monitoring participants for safety and risk must differ from conventional studies where participants and researchers interact face-to-face. Thus, we provide participants with an electronic method in which they can email and report any questions, concerns, or abnormal events they believe to be related to the study, at any time. We also provide participants with a specific avenue within our app to send health-related questions, as opposed to general questions. This allows us to filter and expedite safety and risk-related concerns. If a health concern is submitted via email, a healthcare professional on the research team will contact the participant as soon as possible for additional information and will subsequently inform Mount Sinai of the event.

\subsection{Analysis}

\subsubsection{Individual study performance}

For individual cognition study results, we will use the outcomes of each cognitive test: (a) RAT, (b) Stroop Test, and (c) Trail Making Test. Each test outcome will be analyzed separately. Because both caffeine and L-theanine are short-acting, we do not anticipate carryover effects between treatment periods. This individual analysis uses a linear multiple regression fit and allows us to make a statement of treatment vs. baseline. At present, we are not comparing the treatments to each other.

For each study duration, we will measure the proportion of completed n-of-1 trials that yield statistically meaningful results, for the comparisons (a) caffeine vs. baseline, (b) caffeine + L-theanine vs. baseline. A study is considered complete if a participant reaches 


\section{Measuring the effects of caffeine and L-theanine on cognitive performance: a protocol for}

self-directed, mobile n-of-1 studies

the end of a trial without a study failure or voluntary withdrawal. A study failure occurs when there is insufficient data generated during baseline or any treatment period due to missed treatments (self-reported) or incomplete assessments. Participants must complete $1 / 3$ of treatments and assessments during each treatment period to avoid study failure and involuntary withdrawal. A study will be considered to have yielded a statisticallymeaningful result if the coefficient on the treatment effect is significantly different from zero at the $80 \%$ confidence level in at least one of the three models; that is, if taking caffeine (relative to baseline, with or without L-theanine) produces an effect on cognitive performance measured by at least one of the three cognitive tests. The current confidence level was chosen arbitrarily, with a plan to develop a feature in the future that allows individuals to set their own preferred level of statistical significance.

Analysis of individual cognition studies may change in the future, as we will retain the raw data for each trial. For instance, we may choose to add a comparison of treatments to each other.

Of note, since the study is looking at individual outcomes, the consistency of caffeine and L-theanine dosage across individuals is not an outcome-related concern. Furthermore, the precise quantity of each treatment is not critical to the study design. Individual participant consistency in caffeine and L-theanine intake is the most important treatment factor related to the study outcome.

The code for calculating an individual numerical result will be made publicly available.

\subsubsection{Platform performance}

As previously described, we will also evaluate the performance of the platform across several additional outcome measures: (a) proportion of studies completed, (b) proportion of studies yielding a statistically significant result, and (c) adherence, defined as the proportion of total actions (treatments + assessments) completed by a participant during a study (34)(35). We will use a Bayesian logistic regression model to assess whether the randomized elements of the study (length, notifications) affect study completion, adjusting for any variance in age and sex. Study adherence will be assessed using a Bayesian survival-style model with semi-competing risks over the course of the study.

\subsection{Sharing of individual data and results} Individuals will receive their personalized study results at the end of their study, on the app. They will be presented with graphical, numerical, and textual representations of the results, comparing both treatments to baseline measurements (Fig. 6). Upon completion of the study, participants will also have the option to download their raw data. 
There is a precedent for research participants to dynamically set and adjust their data sharing preferences. Providing the option for global sharing allows participants to contribute to open science and creates increased inferential reproducibility (36). Such dynamic preference-setting is a feature of the mPower Mobile Parkinson Disease Study, led by researchers at Sage Bionetworks (5). Data sharing language in our consent form is similar to that in existing approved protocols, in order to follow this precedent.

Participants may choose to share their daily cognitive assessment scores with friends and others by exporting the data from the app or by saving results images displayed in the app to their phone. Additionally, participants may choose to share their study data with external researchers. This goes into effect once a participant opts in to global sharing in the app settings. Name, contact information, and other directly identifiable information will never be included in externally shared study data.

Figure 6 | Sample of summary results screen

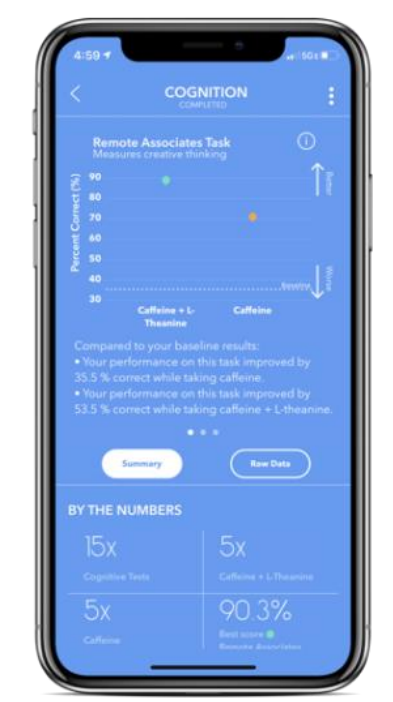

Aggregated study results will be shared with app users via email once published.

\section{Results}

\subsection{Progress to date} participants. This allowed us to fix some general platform issues and address frequent study-related questions, which we subsequently clarified within the app through consent adjustments and the addition of a study-specific FAQ page. We have iterated and improved the N1 app with more than 200 builds over the past two years. 
Measuring the effects of caffeine and L-theanine on cognitive performance: a protocol for self-directed, mobile n-of-1 studies

In October 2019, we fully launched our platform and study on the Apple App Store, with approximately 40 enrolled participants to date. Individual study results will be provided on an ongoing basis, and initial platform performance results will be expected upon completion of approximately 100 studies (34).

\subsection{Ethics approval}

Ethics approval has been requested and granted by the Program for the Protection of Human Subjects at Icahn School of Medicine at Mount Sinai in New York City (IRB-1800343; IRB-18-00789). This study is conducted in accordance with HIPAA regulations.

\subsection{Trial registration}

ClinicalTrials.gov: NCT04056650

https://clinicaltrials.gov/ct2/show/NCT04056650

\section{Discussion}

\subsection{Relevance}

To our knowledge, this is the first attempt to take n-of- 1 investigations outside of the clinic and into the participant's hands. Bringing n-of-1 studies outside of the clinical realm, and ensuring statistical rigor, allows individuals to engage in regulated experiments about everyday health conditions and outcomes of interest on their own terms. Results of individual studies can inform positive lifestyle changes for participants. We hope that the development of this tool, and the introduction of more studies on the platform, will provide individuals with increased agency over their health and allow them to make conscious health-positive decisions that align with their lifestyle.

Success of the platform in wellness-related treatments may further set the stage for its implementation in clinical medicine as an alternative approach to "therapy by trial" for some treatments.

\subsection{Strengths \& limitations}

One of the strengths of this study is that it introduces a novel model of robust selfinvestigation that can be built upon in future research and practice. Additionally, we are using versions of validated NIH toolbox cognitive assessments (modified for digital use instead of on paper), which allows us to be more confident in our potentially actionable results.

Our choice of study and treatments was informed by what would be implemented most smoothly and successfully as a pilot study on the platform. It was also influenced by availability of assessments and ease of access to treatments. The ubiquity of coffee, tea, and over-the-counter supplements makes the study accessible to many individuals. 


\section{Measuring the effects of caffeine and L-theanine on cognitive performance: a protocol for}

self-directed, mobile n-of-1 studies

However, this cognition study introduces a potential limitation due to our choice of Ltheanine as part of a treatment. While commonly available in tea, L-theanine is most widely known within a population of individuals interested in "nootropics", substances believed to enhance cognition. These individuals, due to their existing interest in utilizing cognition-enhancing supplements, may also be inclined toward self-experimentation. We risk losing a generalizable and diverse participant population through treatment choice. We aim to mitigate this by also recruiting through broad-audience websites and social media. While this potential lack of diversity will not affect individual study results, it may impact the generalizability of our platform performance results if the primary users are not representative of the general population.

\subsection{Implications}

In this study protocol, we described the methods for developing and launching a digital, remote $\mathrm{n}$-of- 1 study. To our knowledge, this study is the first of its kind. It employs a rigorous statistical model that accounts for many variables, bias, and learning effects. The results of the study are expected to be relevant to individual participants who want to make positive lifestyle changes, as well as clinicians and researchers interested in exploring n-of-1 methodology. N1 platform and study implementation can be enhanced by learning about what draws people toward self-investigation and behavior change, as well as what causes digital study drop-out. We anticipate that these insights will become clearer as our cognition study progresses.

\section{$5 \quad$ Conflict of Interest}

All authors declare that the submitted work was carried out without the presence of any personal, professional or financial relationships that could potentially be construed as a conflict of interest.

\section{Author Contributions}

EG wrote the manuscript with support from JB, MJJ, MRJ, and NZ. NZ conceived the original idea. NZ and JB designed the project with support from all authors. EG developed the regulatory framework and is program manager for the project. RV created the design framework for the project and MJJ and MRJ carried out the development. MJJ created the front-end of the platform and MRJ built the back-end. NZ developed the analytical methods. NZ and JB directed the project.

\section{$7 \quad$ Funding}

This project was fully supported by internal funding from the Icahn School of Medicine at Mount Sinai.

\section{Acknowledgments}


571 We thank Julian Salisbury for his generous donation to support the development and success of

572 this project. Additionally, we thank all of our volunteer beta testers and lab members for

573 providing continual feedback and support.

574

\section{Contribution to Field}

576 The growing availability of digital research tools creates a unique opportunity to further develop

577 health research. Individuals are now presented with increased opportunities to participate

578 remotely in research, including going through informed consent processes and interacting with

579 study teams via digital platforms (2). These trends open up the digital research space to the

580 concept of the n-of-1 trial. An n-of-1 trial is typically structured as a single-patient, multiple-

581 crossover comparative effectiveness trial, and is mainly used as a tool for individualized clinical

582 care. To our knowledge, our proposed N1 platform cognition study is the first attempt to take n-

583 of-1 investigations outside of the clinic and into the participant's hands. Bringing n-of-1 studies

584 outside of the clinical realm, and ensuring statistical rigor, allows individuals to engage in

585 regulated experiments about everyday health conditions and outcomes of interest on their own

586 terms. Results of individual studies can inform positive lifestyle changes for participants,

587 allowing for increased agency over their health. 
Measuring the effects of caffeine and L-theanine on cognitive performance: a protocol for

self-directed, mobile n-of-1 studies

589

590

591

592

593

594

595

596

597

598

599

600

601

602

603

604

605

606

607

608

609

610

611

612

613

614

615

616

617

618

619

620

621

622

623

624

625

626

627

628

629

630

631

632

633

634

\section{References}

1. Wang T, Azad T, Rajan R. The Emerging Influence of Digital Biomarkers on Healthcare [Internet]. [cited 2019 Oct 11]. Available from: https://rockhealth.com/reports/theemerging-influence-of-digital-biomarkers-on-healthcare/

2. Mathews SC, McShea MJ, Hanley CL, Ravitz A, Labrique AB, Cohen AB. Digital health: a path to validation. npj Digit Med [Internet]. 2019 Dec 13 [cited 2019 Oct 3];2(1):38. Available from: http://www.nature.com/articles/s41746-019-0111-3

3. U.S. Department of Health and Human Services. Use of Electronic Informed Consent: Questions and Answers [Internet]. Tel; 2016 [cited 2019 Oct 3]. Available from: https://www.fda.gov/media/116850/download

4. Home / Usability.gov [Internet]. [cited 2019 Nov 15]. Available from: https://www.usability.gov/

5. Bot BM, Suver C, Neto EC, Kellen M, Klein A, Bare C, et al. The mPower study, Parkinson disease mobile data collected using ResearchKit. Sci Data [Internet]. 2016 Dec 3 [cited 2019 Oct 3];3(1):160011. Available from: http://www.nature.com/articles/sdata201611

6. Turakhia MP, Desai M, Hedlin H, Rajmane A, Talati N, Ferris T, et al. Rationale and design of a large-scale, app-based study to identify cardiac arrhythmias using a smartwatch: The Apple Heart Study. Am Heart J [Internet]. 2019 Jan 1 [cited 2019 Oct 3];207:66-75. Available from: https://www.sciencedirect.com/science/article/pii/S0002870318302710?via\%3Dihub

7. Apple launches three innovative studies today in the new Research app - Apple [Internet]. [cited 2019 Nov 15]. Available from: https://www.apple.com/newsroom/2019/11/applelaunches-three-innovative-studies-today-in-the-new-research-app/

8. Kravitz R, Duan N, Eds, and the DEcIDE Methods Center N-of-1 Guidance Panel (Duan N, Eslick I, Gabler NB, Kaplan HC, Kravitz RL, Larson EB, Pace WD, Schmid CH, Sim I VS. Design and Implementation of N-of-1 Trials: A User's Guide [Internet]. AHRQ Publication No. 13(14)-EHC122-EF. Rockville, MD: Agency for Healthcare Research and Quality; 2014 [cited 2019 Oct 3]. Available from: https://effectivehealthcare.ahrq.gov/products/n-1-trials/research-2014-4/

9. Reynolds AN, Mann JI, Williams S, Venn BJ. Advice to walk after meals is more effective for lowering postprandial glycaemia in type 2 diabetes mellitus than advice that does not specify timing: a randomised crossover study. Diabetologia [Internet]. 2016 Dec 17 [cited 2019 Oct 3];59(12):2572-8. Available from: http://link.springer.com/10.1007/s00125-016-4085-2

10. Nikles CJ, Mitchell GK, Del Mar CB, Clavarino A, McNairn N. An n-of-1 trial service in clinical practice: testing the effectiveness of stimulants for attention-deficit/hyperactivity disorder. Pediatrics [Internet]. 2006 Jun 1 [cited 2019 Oct 3];117(6):2040-6. Available from: http://www.ncbi.nlm.nih.gov/pubmed/16740846

11. Weintraub S, Dikmen SS, Heaton RK, Tulsky DS, Zelazo PD, Bauer PJ, et al. Cognition assessment using the NIH Toolbox. Neurology [Internet]. 2013 Mar 12 [cited 2019 Oct 8];80(11 Suppl 3):S54-64. Available from: http://www.ncbi.nlm.nih.gov/pubmed/23479546

12. ResearchKit [Internet]. [cited 2019 Nov 15]. Available from: http://researchkit.org/

13. Smith A. Effects of caffeine on human behavior. Food Chem Toxicol [Internet]. 2002 Sep 1 [cited 2019 Oct 3];40(9):1243-55. Available from: 
Measuring the effects of caffeine and L-theanine on cognitive performance: a protocol for self-directed, mobile n-of-1 studies

635 https://www.sciencedirect.com/science/article/pii/S0278691502000960

14. Haskell CF, Kennedy DO, Milne AL, Wesnes KA, Scholey AB. The effects of 1-theanine, caffeine and their combination on cognition and mood. Biol Psychol [Internet]. 2008 Feb 1 [cited 2019 Oct 3];77(2):113-22. Available from: https://www.sciencedirect.com/science/article/pii/S0301051107001573?via\%3Dihub

15. Food and Drug Administration. GRAS Notice 000338: L-theanine [Internet]. [cited 2019 Oct 3]. Available from: http://www.fda.gov/Food/FoodIngredientsPackaging/GenerallyRecognizedasSafeGRAS/ GRASListings/default.htm

16. Bryan J. Psychological effects of dietary components of tea: Caffeine and L-theanine. Vol. 66, Nutrition Reviews. 2008. p. 82-90.

17. Keenan EK, Finnie MDA, Jones PS, Rogers PJ, Priestley CM. How much theanine in a cup of tea? Effects of tea type and method of preparation. Food Chem [Internet]. 2011 Mar 15 [cited 2019 Oct 11];125(2):588-94. Available from: https://www.sciencedirect.com/science/article/pii/S0308814610011416

18. Mednick SA. The Remote Associates Test*. J Creat Behav [Internet]. 1968 Jul 1 [cited 2019 Oct 3];2(3):213-4. Available from: http://doi.wiley.com/10.1002/j.21626057.1968.tb00104.x

19. Dallob PI, Dominowski RL. Erroneous solutions to verbal insight problems: Effects of highlighting critical material. 1993.

20. Schooler J, Melcher J. The ineffability of insight. In: Smith SM, Ward TB, Finke RA, editors. The Creative Cognition Approach. MIT Press. 1995;97-133.

21. Bowden EM, Jung-Beeman M. Normative data for 144 compound remote associate problems. Behav Res Methods, Instruments, Comput [Internet]. 2003 Nov [cited 2019 Oct 3];35(4):634-9. Available from: http://www.springerlink.com/index/10.3758/BF03195543

22. Collection of RAT items | What word relates to all three?|Remote Associates Test of Creativity [Internet]. [cited 2019 Oct 3]. Available from: https://www.remote-associatestest.com/

23. Jensen AR, Rohwer WD. The stroop color-word test: A review. Acta Psychol (Amst) [Internet]. 1966 Jan 1 [cited 2019 Oct 4];25:36-93. Available from: https://www.sciencedirect.com/science/article/pii/0001691866900047

24. Woltz DJ, Was CA. Availability of related long-term memory during and after attention focus in working memory. Mem Cogn. 2006;34(3):668-84.

25. Lessons Learned Implementing ResearchKit for a Study at Mount Sinai [Internet]. [cited 2019 Oct 31]. Available from: http://hd2i.org/blog/2019/07/24/researchkit-forresearch.html

26. Reitan RM. Validity of the Trail Making Test as an Indicator of Organic Brain Damage. Percept Mot Skills [Internet]. 1958 Dec 31 [cited 2019 Oct 4];8(3):271-6. Available from: http://journals.sagepub.com/doi/10.2466/pms.1958.8.3.271

27. Spreen O, Benton AL. Comparative studies of some psychological tests for cerebral damage. J Nerv Ment Dis [Internet]. 1965 May [cited 2019 Oct 4];140(5):323-33. Available from: https://insights.ovid.com/crossref?an=00005053-196505000-00002

28. Lezak, M. D., Howieson, D. B., \& Loring DW. Neuropsychological assessment (3rd ed.). New York Oxford Univ Press. 1995;

29. Kortte KB, Horner MD, Windham WK. The Trail Making Test, Part B: Cognitive Flexibility or Ability to Maintain Set? Appl Neuropsychol [Internet]. 2002 Jun [cited 2019 
Measuring the effects of caffeine and L-theanine on cognitive performance: a protocol for self-directed, mobile n-of-1 studies

Oct 4];9(2):106-9. Available from: http://www.tandfonline.com/doi/abs/10.1207/S15324826AN0902_5

30. Lamberty, G. J., Putnam, S., Chatel, D., Bieliauskas, L., \& Adams KM. Derived trail making test indices: A preliminary report. Neuropsychiatry, Neuropsychol Behav Neurol. 1994;7(3):230-234.

31. Arbuthnott K, Frank J. Trail Making Test, Part B as a Measure of Executive Control: Validation Using a Set-Switching Paradigm. J Clin Exp Neuropsychol [Internet]. 2000 Aug 9 [cited 2019 Oct 4];22(4):518-28. Available from: https://www.tandfonline.com/doi/full/10.1076/1380-3395\%28200008\%2922\%3A4\%3B1$0 \% 3 \mathrm{BFT} 518$

32. Caffeine. [cited 2019 Oct 3]; Available from: https://medlineplus.gov/caffeine.html

33. Doerr M, Suver C, Wilbanks J. Developing a Transparent, Participant-Navigated Electronic Informed Consent for Mobile-Mediated Research. SSRN Electron J. 2017 Jun 28;

34. Bobe J, Buros J, Golden E, Johnson M, Jones M, Percha B, et al. Striking the Right Balance: a protocol to evaluate factors associated with completion and adherence in appbased n-of-1 trials. JMIR Prepr [Internet]. 2019 [cited 2019 Oct 10]; Available from: https://preprints.jmir.org/preprint/16362

35. Assessing the Effectiveness of an N-of-1 Platform Using Study of Cognitive Enhancers [Internet]. [cited 2019 Oct 22]. Available from: https://clinicaltrials.gov/ct2/show/NCT04056650?term=NCT04056650\&rank=1

36. Plesser HE. Reproducibility vs. Replicability: A brief history of a confused terminology. Front Neuroinform. 2018 Jan 18;11. 
Measuring the effects of caffeine and L-theanine on cognitive performance: a protocol for self-directed, mobile n-of-1 studies

\section{Appendix A: Cognition Assessment Details}

\section{Remote Associates Test (RAT)}

This test was implemented as a variation of the Remote Associates Test, originally designed by Mednick in 1962, and developed as a custom digital ActiveTask within Apple's ResearchKit framework (18). Based on the duration of some trials in our cognition study, a greater number of problems were needed than were presented in the original RAT. As a result, a total of 312 word problems were sourced from an online database with an expanded inventory of items (22). For each test, a total of 10 "questions" is presented to the individual. Each question represents one of the word problems, where each word problem is chosen randomly, and will never be subsequently repeated for the individual. For each question, the three stimulus words are presented simultaneously in a normal horizontal orientation at the center of the screen and the user has a 30 second time limit for completion. At the top of the screen, a label is presented indicating the current question number, i.e. "Question 1/10". Just below this label, a timer is presented that would count down from 30 seconds. An onscreen keyboard also appears to allow the individual to type their solution, which is visible in the textbox below the three stimulus words. Just above the onscreen keyboard, two buttons are presented: the first, titled "Submit", is used to submit the proposed solution; and the second, titled "Skip", is used to allow the individual to skip the question if they cannot come up with a solution.

\section{Stroop Test}

The original paper version of this test has 3 tasks: "word", "color", and "color-word." The intent of the original paper test was to compare a difference in mean response time with and without "intervention" (mismatching the word and color) (23). The existing ResearchKit version uses two separate tests that measure response time with the "intervention."

\section{Trail Making Test (TMT)}

The original Trail Making Test consists of two parts: Part A requires the participant to connect 25 dots labeled with numbers in ascending order as quickly as possible. Part B requires that the participant connect dots labeled with letters and numbers, alternating between the two in ascending order (ex. 1-A-2-B-...).

In the predefined ActiveTask, the dots are generated randomly on the screen where the entirety of each dot is visible on the screen. Similarly, there are constraints in dot placement that prevent any lines that correctly connect the labeled dots from crossing. Should the participant make an error, no line is drawn indicating a mistake. An existing label that presents the participant with elapsed time and the number of errors during the test is hidden to prevent the potential influence on current and future performance that may result from displaying the participant's results during an ongoing study. 
Measuring the effects of caffeine and L-theanine on cognitive performance: a protocol for self-directed, mobile n-of-1 studies

\section{Appendix B: Data Categories \& Variables}

\section{Data categories Data variables}

\begin{tabular}{|c|c|}
\hline $\begin{array}{l}\text { App registration } \\
\text { *stored separately from } \\
\text { study-specific data }\end{array}$ & Email, gender, year of birth, password \\
\hline Pre-consent eligibility & $\begin{array}{l}\text { Over } 18(\mathrm{Y} / \mathrm{N}) \\
\text { Pregnancy/Breastfeeding status }(\mathrm{Y} / \mathrm{N}) \\
\text { Caffeine contraindication }(\mathrm{Y} / \mathrm{Not} \text { sure/N) }\end{array}$ \\
\hline Cognitive assessments & $\begin{array}{l}\text { Date/Time } \\
\text { Scores at baseline, Treatment A, and } \\
\text { Treatment B for RAT, Stroop Test, and } \\
\text { TMT } \\
\text { Order of assessments }\end{array}$ \\
\hline Study design & $\begin{array}{l}\text { Treatment choice \& time (e.g. beverage or } \\
\text { supplement; time of day to take treatment) } \\
\text { Length of study } \\
\text { Order of treatments } \\
\text { App notification frequency (light, } \\
\text { moderate) }\end{array}$ \\
\hline Compliance & $\begin{array}{l}\text { \# of missed assessments } \\
\text { \# of missed treatments }\end{array}$ \\
\hline Self-report & $\begin{array}{l}\text { Notes of events \& treatment non- } \\
\text { adherence }\end{array}$ \\
\hline Post-study (optional) & $\begin{array}{l}\text { Anonymous } 10 \text {-item exit survey } \\
\text { (platform-specific feedback) }\end{array}$ \\
\hline
\end{tabular}

